\title{
TRANSFORMATION OF NATIVE DEFECTS IN GaAs UNDER ULTRASONIC TREATMENT
}

\author{
A. MąKosa, T. WosińsKI \\ Institute of Physics, Polish Academy of Sciences \\ Al. Lotników 32/46, 02-668 Warszawa, Poland
}

AND Z. WITCZAK

High Pressure Research Center, Polish Academy of Sciences

Sokolowska 29, 01-142 Warszawa, Poland

\begin{abstract}
Effect of high-intensity ultrasonic vibration on the spectrum of deep electron traps in bulk GaAs has been studied giving rise to a discussion on microscopic structure of native defects associated with the traps.
\end{abstract}

PACS numbers: $61.72 . \mathrm{Ji}, 62.80 .+\mathrm{f}, 71.55 . \mathrm{Eq}$

More than a dozen of various deep electron traps, commonly observed in as-grown GaAs single crystals, were characterized and catalogued already fifteen years ago [1]. Over the years the traps have been intensively studied showing that most of them are associated with native lattice defects, although their atomic structure has not been so far unambiguously resolved. Aimed at obtaining some new information on the structure of these traps we have investigated an effect of high-intensity ultrasonic vibration on the spectrum of deep electron traps, revealed with the deep level transient spectroscopy (DLTS), in bulk GaAs.

The investigated samples were prepared from $n$-type horizontal Bridgman (HB) grown single crystal with the electron concentration of $3 \times 10^{16} \mathrm{~cm}^{-3}$. Three (001) oriented samples, labeled $A, B$ and $C$, were cut in a form of round slabs of $500 \mu \mathrm{m}$ thickness and $10 \mathrm{~mm}$ in diameter. The sample $A$ was considered as a reference one, and the samples $B$ and $C$ were subjected to ultrasonic vibration in an ultrasonic resonator combined with a high-pressure vessel. The vibration at a frequency of $17.5 \mathrm{kHz}$ was applied for $600 \mathrm{~s}$ at a temperature of $0^{\circ} \mathrm{C}$. The ultrasonic experiment was performed under a hydrostatic pressure of $1 \mathrm{GPa}$ in order to avoid the brittle behaviour of $\mathrm{GaAs}$ at low temperature. Two amplitudes of vibration giving rise to the normal stresses in the samples of $100 \mathrm{MPa}$ (sample $B$ ) and $200 \mathrm{MPa}$ (sample $C$ ) were applied.

After the ultrasonic treatment several gold discs of $1 \mathrm{~mm}$ diameter were evaporated onto each sample to form Schottky diodes. The electron concentration in the samples $B$ and $C$ was only slightly affected by the ultrasound, as obtained from the capacitance vs. voltage measurements of the diodes. On the other hand, 
drastic changes in the DLTS spectra of the diodes were revealed as a result of the ultrasonic treatment.

Three deep electron traps, labeled EL2, EL3 and EL6 according to Ref. [1], were revealed in the DLTS spectra of the reference (as-grown) sample, as shown in Fig. 1 (diode \#1). In the samples subjected to the vibration the concentration

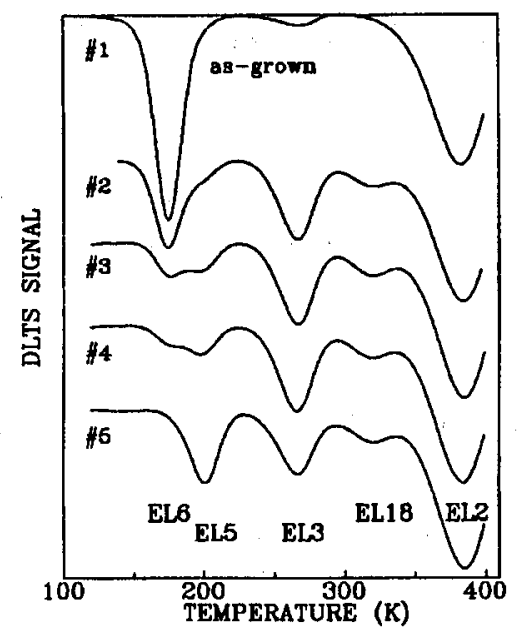

Fig. 1. DLTS spectra of the reference sample $A$ (diode \#1) and the samples $B$ (diodes $\# 2$ and \#3) and $C$ (diodes \#4 and \#5) subjected to ultrasonic treatment.

of the EL6 traps was strongly reduced and two new traps, EL5 and EL18, appeared, and the concentration of the EL3 traps increased. The trap concentrations measured in various diodes of the samples $B$ and $C$ were dependent on the diode position on the sample area, whereas the traps were homogeneously distributed over an area of the reference sample.

In Fig. 1 DLTS spectra of four diodes of the samples $B$ and $C$, numbered from 2 to 5 , are presented. The diodes \#3 and \#5 are placed in the centers of the samples $B$ and $C$, respectively, and the diodes \#2 and \#4 are representative for a half-way between the center and periphery of the samples $B$ and $C$, respectively. The inhomogeneity of the trap concentrations over the samples $B$ and $C$ was considered as a result of an inhomogeneous axially symmetrical distribution of ultrasonic stress in the resonator, being the highest at the center of a sample and the lowest at its periphery.

In Fig. 2 the concentrations of deep traps are presented as a function of the diode number, where the diode \#1 represents the reference sample and the numbers from 2 to 5 correspond to the diodes subjected to increasing amplitude of the ultrasonic vibration. Only the EL2 concentration was not affected by the ultrasonic treatment. The EL6 concentration decreased monotonically with increasing ultrasonic stress applied, and the concentrations of three other traps, EL5, EL3 and EL18, increased at its expense. At the maximum stress used (diode \#5) the EL6 concentration was reduced below the detection limit and the concentrations 


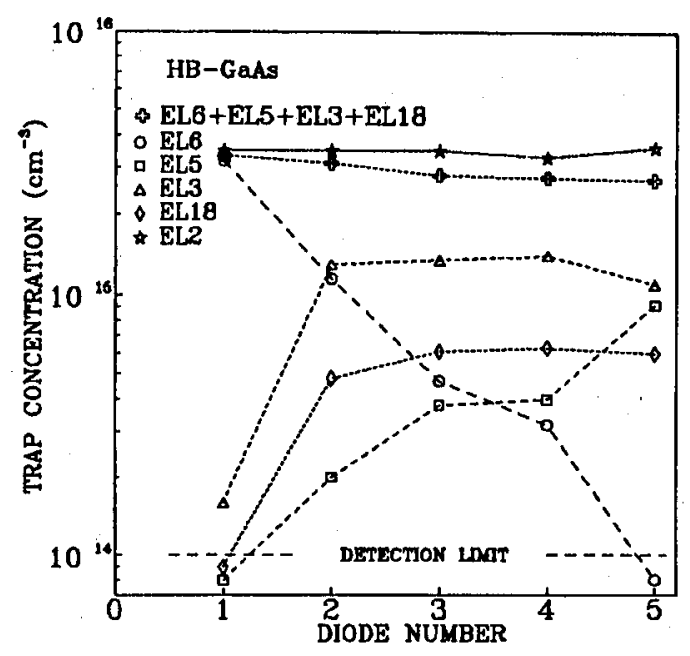

Fig. 2. Concentrations of deep traps measured with DLTS in the reference diode (number 1) and the diodes subjected to increasing amplitude of the ultrasonic vibration (numbers 2 to 5 ).

of the other traps were increased so that the total trap concentration remained almost unchanged. The results point out to a transformation of the EL6 traps into the EL5, EL18 and (partly) EL3 traps driven by the ultrasonic vibration.

Taking into account the present findings together with the data reported so far in the literature, we may speculate on the microscopic structure of the defects associated with the observed traps. The detailed discussion is beyond the scope of this communication and only the main conclusions will be presented below.

The EL2 trap, being the most studied native trap in GaAs, is still a matter of controversy (for recent review see [2]), however, there is now a growing conviction that an isolated arsenic antisite, $\mathrm{As}_{\mathrm{Ga}_{a}}$, is responsible for this trap. The very recent theoretical calculations, being in favour of this identification, are presented in [3].

The EL6 trap, like EL2, is related to excess arsenic. Also a similarity between optical properties of EL2 and EL6 was pointed out [4] and a complex defect involving $\mathrm{As}_{\mathrm{Ga}}$ was proposed to be associated with $\mathrm{EL} 6$ [5]. To explain our present results we postulate a complex $\mathrm{As}_{\mathrm{Ga}}-\mathrm{V}_{\mathrm{As}}$ (As antisite-As vacancy) to be responsible for EL6, which can arise from the nearest-neighbour hop of an As atom onto a Ga vacancy.

The EL3 trap has been recently identified as associated with an oxygen related defect, off-centre $O_{A s}$, exhibiting a negative- $U$ property $[6,7]$.

Further, we postulate that the defects responsible for the EL5 and EL18 traps are a divacancy complex, $\mathrm{V}_{\mathrm{Ga}}-\mathrm{V}_{\mathrm{As}}$, and a $\mathrm{Ga}$ vacancy, $\mathrm{V}_{\mathrm{Ga}}$, respectively. The energy levels of these defects, calculated theoretically using the self-consistent Green's-function technique by Baraff and Schlüter [8], are in accordance with the thermal activation energies obtained for the above traps from DLTS measure- 
ments.

Finally, transformation of the EL6 traps into three other traps occurring under the ultrasonic treatment can be explained by the following defect reactions:

$$
\begin{aligned}
& A s_{G a}-V_{A s} \rightarrow V_{G a}-V_{A s}+A s_{i}, \\
& A s_{G a}-V_{A s}+O_{i} \rightarrow O_{A s}+V_{G a}+A s_{i} .
\end{aligned}
$$

The EL5 traps are produced in the first reaction and both the EL3 and EL18 traps are produced in the latter one, where a limiting factor is the concentration of oxygen interstitials, $\mathrm{O}_{\mathrm{i}}$, in the crystal, which are not electrically active in GaAs. Arsenic interstitials, $\mathrm{As}_{\mathrm{i}}$, which are produced in both reactions are highly mobile, even at room temperature, and can diffuse to sinks, such as the sample surface and dislocations, during the ultrasonic experiment.

In conclusion, taking advantage of the observed transformation of the EL6 trap into three other traps in GaAs under ultrasonic treatment we have discussed microscopic nature of these traps in terms of the native defect complexes formed in crystals grown under $\Lambda$ s-rich conditions.

\section{References}

[1] G.M. Martin, A. Mitonneau, A. Mircea, Electron. Lett. 13, 191 (1977).

[2] G.A. Baraff, Acta Phys. Pol. A 82, 599 (1992).

[3] Q.-M. Zhang, J. Bernholc, Phys. Rev. B 47, 1667 (1993).

[4] A. Chantre, G. Vincent, D. Bois, Phys. Rev. B 23, 5335 (1981).

[5] M. Levinson, Inst. Phys. Conf. Ser. 91, 73 (1988).

[6] M. Skowronski, S.T. Neild, R.E. Kremer, Appl. Phys. Lett. 57, 902 (1990).

[7] U. Kaufmann, E. Klausmann, J. Schneider, H.Ch. Alt, Phys. Rev. B 43, 12106 (1991).

[8] G.A. Baraff, M. Schlüter, Phys. Rev. Lett. 55, 1327 (1985); Phys. Rev. B 33, 7346 (1986). 